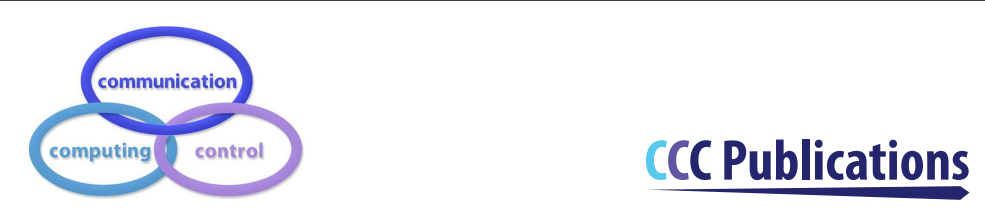

\title{
A Fuzzy Logic Architecture for Rehabilitation Robotic Systems
}

\author{
Y. Bouteraa, I.B. Abdallah, A. Elmogy, A. Ibrahim, U. Tariq, T. Ahmad
}

\author{
Yassine Bouteraa* \\ 1. Department of Computer Engineering, Prince Sattam Bin Abdulaziz University, KSA \\ 2. Digital Research Center of Sfax \& CEM Lab-ENIS, University of Sfax, Tunisia \\ *Corresponding author: y.bouteraa@psau.edu.sa
}

\section{Ismail Ben Abdallah}

Digital Research Center of Sfax \& CEM Lab-ENIS, University of Sfax, Tunisia ismail.benabdallah@enis.tn

\author{
Ahmed Elmogy \\ 1. Department of Computer Engineering, Prince Sattam Bin Abdulaziz University, KSA \\ 2. Computers \& Control Eng. Department, Faculty of Engineering, Tanta University, Egypt \\ a.elmogy@psau.edu.sa
}

\section{Atef Ibrahim \\ Department of Computer Engineering, Prince Sattam Bin Abdulaziz University, KSA aa.mohamed@psau.edu.sa}

\section{Usman Tariq, Tariq Ahmad}

Department of Information Systems, Prince Sattam Bin Abdulaziz University, KSA

u.tariq@psau.edu.sa, t.ahanger@psau.edu.sa

\begin{abstract}
Robots are highly incorporated in rehabilitation in the last decade to compensate lost functions in disabled individuals. By controlling the rehabilitation robots from far, many benefits are achieved. These benefits include but not restricted to minimum hospital stays, decreasing cost, and increasing the level of care. The main goal of this work is to have an effective solution to take care of patients from far. Tackling the problem of the remote control of rehabilitation robots is undergoing and highly challenging. In this paper, a remote wrist rehabilitation system is presented. The developed system is a sophisticated robot ensuring the two wrist movements (Flexion / extension and abduction/adduction). Additionally, the proposed system provides a software interface enabling the physiotherapists to control the rehabilitation process remotely. The patient's safety during the therapy is achieved through the integration of a fuzzy controller in the system control architecture. The fuzzy controller is employed to control the robot action according to the pain felt by the patient. By using fuzzy logic approach, the system can adapt effectively according to the patients' conditions. The Queue Telemetry Transport Protocol (MQTT) is considered to overcome the latency during the human robot interaction. Based on a Kinect camera, the control technique is made gestural. The physiotherapist gestures are detected and transmitted to the software interface to be processed and be sent to the robot. The acquired measurements are recorded in a database that can be used later to monitor patient progress during the treatment protocol. The obtained experimental results show the effectiveness of the developed remote rehabilitation system.
\end{abstract}

Keywords: Rehabilitation robotics, fuzzy logic, vision-based gesture control, IoT. 


\section{Introduction}

In recent years, special attention has been paid to the integration of robots in health systems, mainly in rehabilitation and surgery applications [20,43]. Rehabilitation is care that is mainly used to restore or improve the functions of diminished parts of stroked or disabled individuals. This in turn will help these individuals to return partially or completely to their active lifestyle. Physical rehabilitation is the most popular type of rehabilitation. The physical rehabilitation can be achieved conventionally through the physical interaction between the patients and therapists. However, this is ineffective as it is costly, and time consuming. On the other hand, the coupling of the great advances in robotic systems and computers made a paradigm shift in the medical rehabilitation especially in the last decade. Thus, incorporating robots and computers in the rehabilitation process is a must as it highly increases the effectiveness of the whole process, reduces costs, and encourages patients to be rehabilitated. Examples of rehabilitation robotic devices are wearable robots, robotic orthoses, and robotic wheelchairs. Most of these devices are either concerned with the rehabilitation of lower or upper limb [16, 22, 28].

The utilization of robots in rehabilitation is very challenging as it requires looking for solutions that address several patients' capabilities. Semi or fully autonomous robots can be adopted for this purpose. The main motivation of using robots in rehabilitation is the high increase of the number of elderly and disabled people. The research efforts of involving robots in rehabilitation field led to the development of many interesting and novel rehabilitation devices. A summary of these devices can be found in [32]. Working towards developing such type of devices requires a deep recognition and special skills to solve many mechanical, electrical, biological, and software issues. Great research efforts have been working to solve these issues $[16,28,32]$. Many benefits can be gained from using robots in rehabilitation starting from the repetition of exercises and ending with the complete monitoring of patients during the exercise protocol using the robot sensors [5].

On the other hand, the Internet of Things (IoT) has recently been used in many research areas and applications $[35,36]$. By using IoT concept, the system parts/objects can be accessed anytime/anywhere [47]. By using IoT concept, monitoring and control of the whole system is achieved effectively and efficiently. Internet of robotics is one of the most important concepts that integrates robotics with IoT to overcome the limitations of cloud robotics [40]. Using the internet of robotics concept paved the way to control the rehabilitation robots remotely which in turn will greatly affect the whole rehabilitation process and all stakeholders [27]. The tele-rehabilitation systems will allow the therapists to remotely control and monitor their patients at home. Many human robot interactions approaches and interfaces have been developed to tackle the challenges of the tele-manipulation problem [6, 25, 26, 31, 44].

Fuzzy logic has been extensively used in many applications [12, 18, 33, 39]. This mainly comes from the powerfulness of fuzzy logic in dealing with uncertain and inaccurate data. In [33], the authors combine the characteristics of the adaptive Nertwork-based Fuzzy Inference System (ANFIS) and Ordinary Differential Equations (ODEs) in one model able to represent the genetic regulatory networks (GRN). In [39], a fuzzy logic control algorithm based on a Takagi-Sugeno-Kang inference engine is proposed to stabilize the Rossler chaotic dynamical system. The Professor Zadehs' contributions to the development of Soft Computing, and Artificial Intelligence along their influences on the world and Romania are summarized in [12]. A fuzzy model and a methodology to determine the optimal green period ratios of a traffic light is presented in [18].

Many researches have focused on fuzzy logic based control schemes for rehabilitation robots. An adaptive admittance controller based on fuzzy logic has been developed for 2-DOF actuated parallel ankle rehabilitation robot [41]. Also, a PID controller based on a fuzzy inference system for rehabilitation of shoulder flexion/extension has been presented in [3]. Additionally, a fuzzy-impedance control law is developed to estimate the human-robot interaction force in [51]. A robotic fuzzy-based adaptive assistance system is presented in [21]. In contrast to these works which adopt fuzzy logic either as a control law for trajectory tracking $[3,41]$ or for assistance $[21,51]$, the proposed solution in this paper integrates the concept of fuzzy logic into the adaptive control architecture to build a safety system that takes pain that could be felt by patients into account.

The fuzzy-logic-based decision-making approach are discussed in various works $[13,14,19,45,48]$. 
In [19], an adaptive impedance force controller is developed for an upper limb rehabilitation robot. The fuzzy logic regulator is used to make decision about the estimated force to be generated to suit the muscular strength of the affected limb. In [45], authors focus on the risk assessment of a robotic system dedicated to the rehabilitation of the elbow and wrist. The risk assessment is analyzed using a system based on fuzzy logic which confirms whether the robot is secure or not and determines this degree of security. In [48], the control based on the RLS algorithm (Recursive Least Square) is developed to identify the patient's residual motor capacity. Depending on the impedance of the upper limb in motion, the reference trajectory is generated according to the patterns of movement in healthy humans. In addition, the fuzzy logic control strategy is implemented in the direction perpendicular to the movements making it possible to make the right decision on the level adjustment of the robotic assistance while taking into account the impedance of the upper limb and the variations of deviation between the reference trajectory and real trajectory. A fuzzy logic approach is used in this paper to enable system to effectively adapt to the patient's conditions. Indeed, the solution integrates a current sensor in order to have an indication of the patient's pain. The presence of a current peak can be explained by a resistive force. The resistance force occurs naturally and unconsciously when the patient experiences pain. The fuzzy controller takes these current levels and the last angle into account to estimate whether the felt pain requires stopping the action or not.

On the other hand, the Kinect sensor has shown elegant capabilities making it an excellent option to consider in any vision-based applications. Examples of these applications are assistive systems $[24,38]$, rehabilitation [1, 2, 8, 9, 42], and navigation systems [46, 50]. Also, Kinect has been seen in many gesture recognition approaches $[10,11,17,23]$. A Kinect gesture recognition approach is proposed in this paper to improve the process of implementing gesture recognition. Actually, the developed system is a vision-based remote handling solution which avoids the disadvantages of EMGbased solutions. The Kinect camera is used to recognize gesture movement of the therapist. The recognized movement is transferred through the internet to the wearable robot at the patient side. The use of Kinect is very beneficial for the therapist during the initialization of the gesture as no need for manipulation or explanation. The patient is completely passive and the robot must repeat the action of the physiotherapist. This kind of exercise maintains mobility, and flexibility. In the early stages of the rehab protocol, we are often faced with cases that cannot be active and therefore passive ROM (range of motion) exercises can reduce joint stiffness and prevent it from freezing due to lack of movement.

This paper focuses on the development and implementation of a wrist tele-rehabilitation system that significantly solve the rehabilitation problems. By using the proposed system, the therapists is capable of dealing with many patients at a time which in turn decrease the number of patients in the waiting list of rehabilitation centers. Also, the remote patient monitoring and control capabilities of the proposed system can greatly decrease healthcare costs [27]. Using such type of home rehabilitation systems will help patients to start their therapy protocol quickly which in turn accelerates their recovery. The proposed system is completely considering the safety of patients during the treatment protocol through employing a current sensor that measures the patient pain during the rehabilitation exercises. The developed tele-rehabilitation implements a whole system allowing the physiotherapist to act in a remotely gestural way to control a robot and to have visual feedback. During the study of the control architecture, it has been found that the MQTT protocol is more suitable for the developed application and its constraints than the famous HTTP (Hypertext Transfer Protocol). The most important characteristic of the MQTT protocol is the real time aspect which avoids problems caused due to latency. Additionally, the MQTT protocol is easy to implement and enables fast data transmission by means of publish/subscribe operations, which are essential in medical applications and ideal for machine-to-machine communication. The use of the network must be minimized to avoid disturbances due to the high demand for data exchange. The MQTT protocols minimize the data packets which alleviate the use of the network and consequently avoid disturbances caused by the high demand for data exchange. MQTT is a low-power protocol offering the possibility of using a battery as an alternative solution to power the portable robot.

The main contribution of the presented work is that it integrates the vision-based gesture control, the adaptive control and fuzzy logic approaches and IoT concept in rehabilitation robotic device to 
help therapists to remotely take care of patients efficiently and reliably. In addition, the software developed for the proposed e-rehabilitation system displays real-time performance, augmented reality interface and provides a database table which ensures patient care monitoring and electronic medical records. The proposed IoT rehabilitation robotic system is presented in figure 1. The details of the control design is presented in the next section. The details of the control design are presented in the next section. This includes the adaptive control and fuzzy logic control.

\section{Control design}

Gesture control is the process of recognizing and interpreting movements of human body to control a computer system. This type of interaction between human and computer system is achieved through software interface. Various approaches have been developed in the literature for this purpose [10, 23, 26]. Recently, vision based recognition is used for gesture control to overcome the limitations of using traditional sensors to recognize the required actions. Using conventional $2 \mathrm{D}$ cameras can only provide general information about human movement and thus is not appropriate for many applications. Furthermore, the 2D vision recognition systems are subject to environmental factors that affect the recognition of object movements such as lighting, contrast, and distance. Alternatively, the 3D vision systems have shown excellent capabilities of recognizing object movements [10, 23]. Kinect is one of the amazing 3D cameras that can be used for 3D imaging. The most important feature that makes Kinect an excellent choice for gesture recognition is its depth imaging. This depth imaging feature makes it easy to recognize the distance between the Kinect and the moving object. Kinect is used in this paper for gesture control.

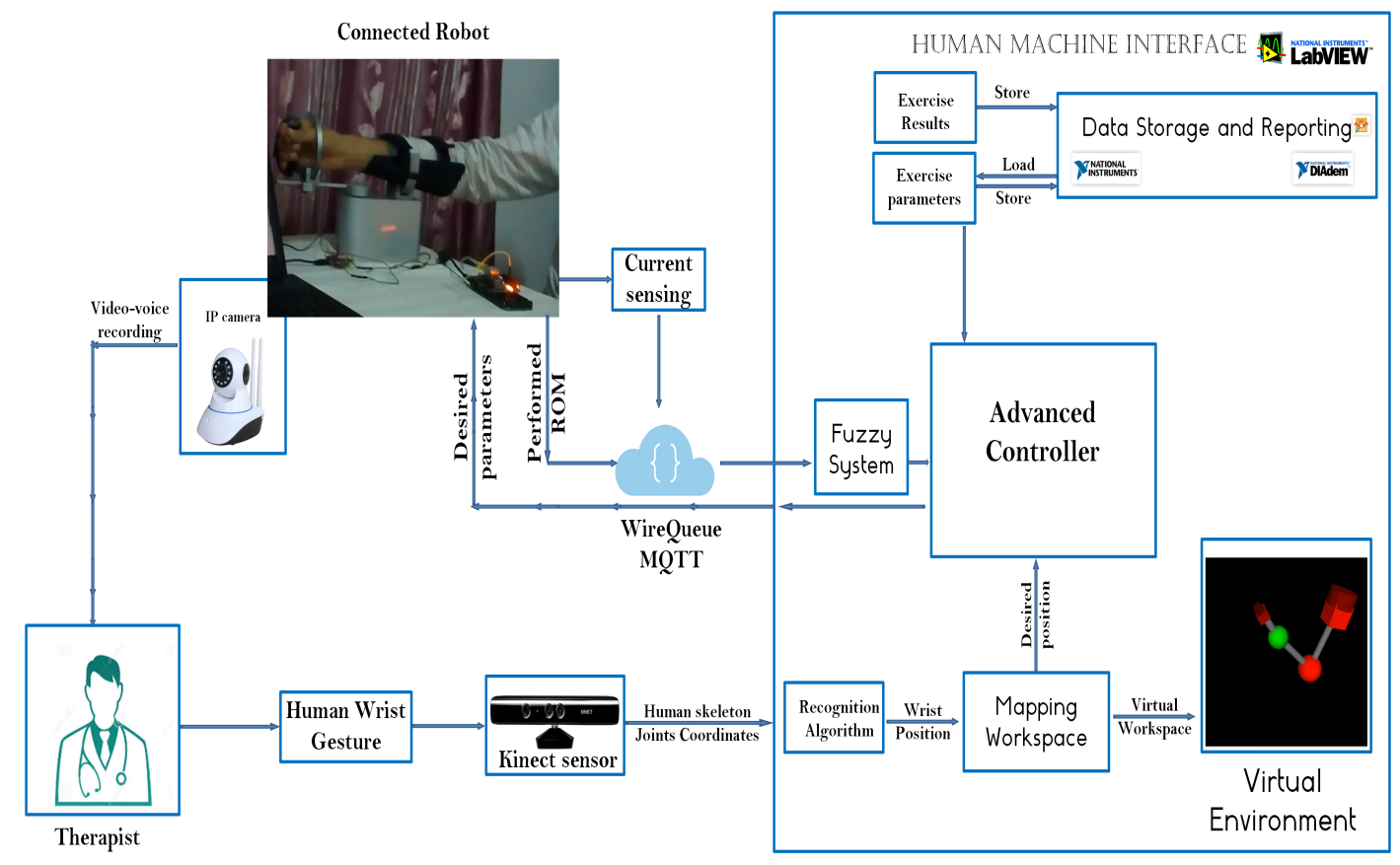

Figure 1: Control Architecture Overview

The developed software is done on the Laboratory Virtual Instrument Engineering Workbench (LabVIEW) environment. By using LabView, we benefit from the ability to integrate user interface design into the development cycle and take advantage of numerous supports allowing easy interfacing with cameras, instruments, boards and other devices. Starting by the physiotherapist as the system operator, the gesture is captured by the Kinect sensor. The signal processing and recognition are run on the computer system (CPU: Intel Core i7, 8th Generation, 8705G / $3.1 \mathrm{GHz}$ ) using the Microsoft Kinect API (Application Programmer Interface) developed by the NI (National Instrument). One of the highlights of the SDK is its very fast and accurate pattern recognition system, which does not require any configuration or calibration of the sensor at the start. In order to identify the exoskeleton, this recognition system compares the presented gesture with all registered models in order to find a 
match. To detect its exoskeleton, it is sufficient for the user to appear just in front of the sensor ensuring that his head and body are visible. Processed data must pass through a mapping block before being used in the control law set point. Since the system is in prototyping phase, the used equipment is based on open source hardware platforms. The Arduino Due board based on 32-bit ARM core microcontroller is used as the embedded system controller. The communication with the server is ensured through a Wi-Fi shield (Yun Shield board). Arduino Due plays the role of the interface board and not the brain of the designed system. The microcontroller receives the real position and transmits it to the pilot. As presented in figure 1, information about the actual position and current sensing are recovered from encoder and the driver circuit, respectively. These data are sent through the server to the computer system to be the inputs of the fuzzy controller. The pain estimation is defined according to the implemented fuzzy rules. In the implemented block of the nonlinear control, a test is done to identify the state coming from the Fuzzy block. Based on this state, the adaptive controller either adopts the desired angle or keeps the previous angle.

Figure 2 shows the detailed steps of the proposed system starting from the therapist and going to the patient and returning again to the therapist through visual feedback. As illustrated in Figure 2, the microcontroller receives the real position and current sensing and transmits them to the physiotherapist station. These data are sent through the server to the computer system to be the inputs of the fuzzy controller. The pain estimation is defined according to the implemented fuzzy rules. The proposed system has the ability to store and report the patient exercise data to monitor the patient progress during the therapy protocol.

The physiotherapist station (running MQTT broker) publishes topics and values to the broker. The robot subscribes to the broker to be notified when these topics are updated. Certain technical points must be considered when comes to the implementation of this protocol. Mosquitto, as an open source message broker is running in the physiotherapist computer, while PubSubClient, as an Arduinobased MQTT client is implemented in the robot embedded system. The physiotherapist uses gesture control to send the joint angle to the server. The physiotherapist station defines the angle value as a topic to publish. On the other side, the Arduino due-based system must subscribe to this topic to receive the transmitted message. The robot also sends feedback to the physiotherapist's computer. In this case, the measured angle is the topic that the physiotherapist computer must subscribe to.

The connection of the Arduino due board to the internet has been developed using the wifi library. This library requires SSID broadcasting and provides data encryption using Wireless Protected Access (WPA2) which is the improved version of the WPA standard. The Arduino Due board communicates with the wifi shield through the SPI bus. The media access control address (MAC address) is provided by the wifi shield and consequently the robot has its physical address as a unique identifier for the network interface controller (NIC). Http request is sent by the physiotherapist interface to the server. On his part, the web server sends a request to the connected robot and establishes a communication with the SQL server to store data. The connection between LabVIEW interface and the SQL database is based on LabVIEW database connectivity toolkit. The embedded system in the wrist robot receives the desired angles, operates and sends feedback.

\subsection{Adaptive control}

In general, the adaptation of a system to its environment lies in the possibility of reacting to the variations that this environment may undergo. In control theory, adaptive control aims to react to variations in the system. In the non-adaptive approach, the development of control algorithms is done by considering an invariant model. However, in the case of systems whose parameters are unknown, sophisticated control approaches such as adaptive control are applied to deal with this model uncertainty. Within the framework of our application and in addition to the uncertainty of the robot model, working with arms of different weights confirms this uncertainty of the dynamic model of the system. Consequently, we use this adaptive approach in order to real-time react by minimizing the error between the set point and the output even in the presence of system variations.

The developed architecture uses one link robot which can be modeled as follows:

$$
m(q) \ddot{q}+b \dot{q}+g(q)=\tau
$$




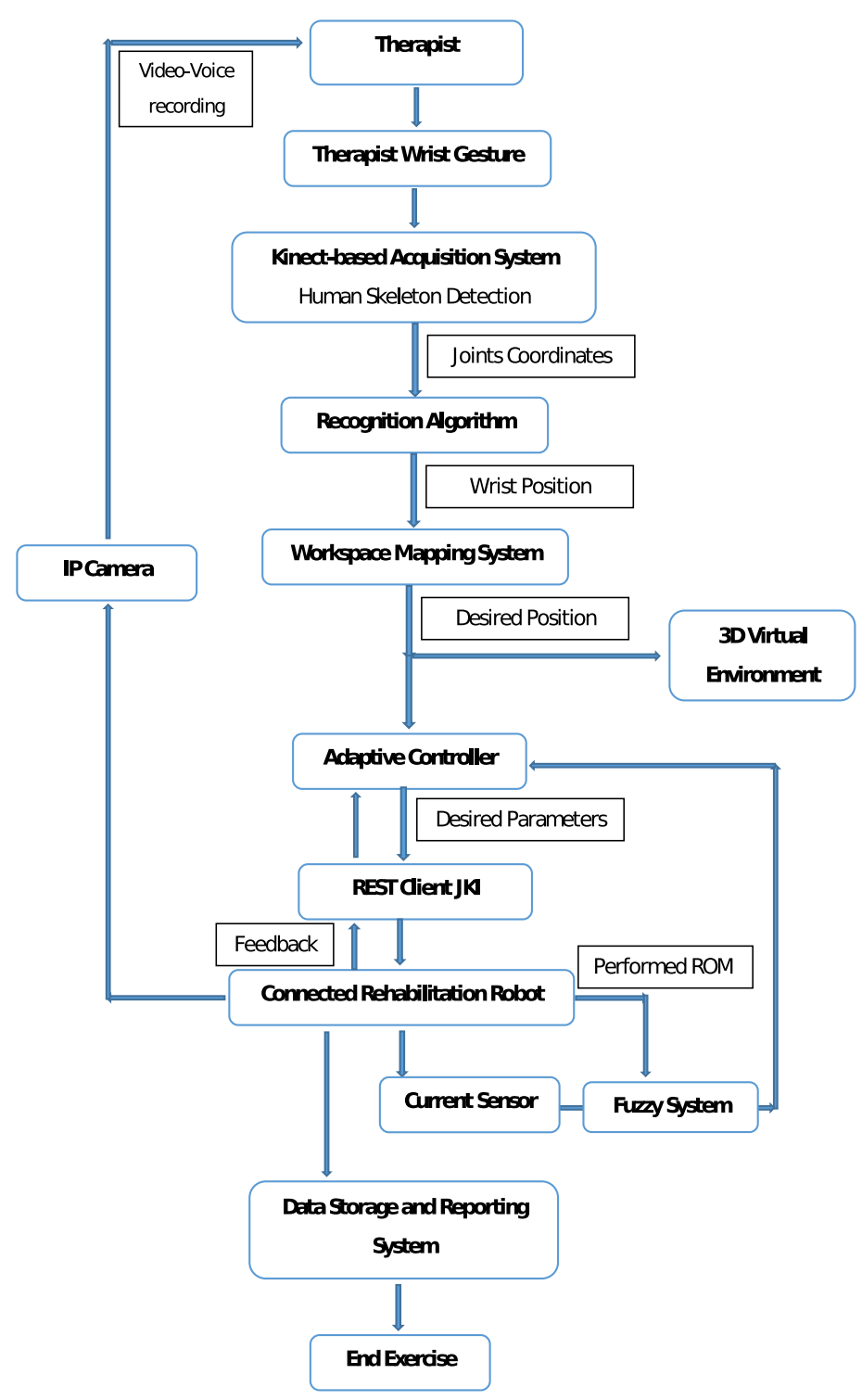

Figure 2: Flowchart of the implemented system

where

$m$ is the mass of the robot link

$q$ is the joint variable

$\dot{q}$, and $\ddot{q}$ are the velocity and acceleration of the robot joint respectively

$b$ is a positive scalar representing the dynamic coefficient of friction

$g$ represents the gravitational force

Equation 1 can be written as:

$$
m \dot{r}=y \Phi-\tau-b r
$$

where

$$
r=\lambda e+\dot{e}
$$

$e=q_{d}-q$ represents the tracking error, $q_{d}$ is the desired trajectory of the joint variable, and $\lambda>0$.

$$
y \Phi=m\left(\ddot{q}_{d}+\lambda \dot{e}\right)+b\left(\dot{q}_{d}+\lambda e\right)+g(q)
$$

where $y$ is the regression matrix

$y=\left[\begin{array}{ll}y_{11} & y_{12}\end{array}\right]$

$\phi$ is $2 x 1$ vector of the unknown constant parameters 
The following controller is proposed:

$$
\tau=y \hat{\phi}+k_{v} r
$$

where $k_{v}$ is a scalar control gain

With the adaptive update rule:

$$
\dot{\hat{\phi}}=-\dot{\tilde{\phi}}=\gamma y^{T} r
$$

where

$\hat{\phi}$ is the estimate vector of the unknown parameters

$\hat{\phi}$ is $2 x 1$ matrix $\left(\hat{\phi} \in \Re^{2 x 1}\right), \tilde{\phi}=\phi-\hat{\phi}$

$\gamma=\left(\begin{array}{cc}\gamma_{1} & 0 \\ 0 & \gamma_{2}\end{array}\right)$, and $\gamma_{i}>0$.

In order to prove the stability of the proposed controller, the Lyapunov function $\mathrm{V}$ can be written as:

$$
\begin{aligned}
& V=\frac{1}{2} r^{T} m(q) r+\frac{1}{2} \tilde{\phi^{T}} \gamma^{-1} \phi^{2} \\
& \dot{V}=r^{T} \dot{r}+\frac{1}{2} r^{T} \dot{m} r+\tilde{\phi^{T}} \gamma^{-1} \tilde{\dot{\phi}}
\end{aligned}
$$

Substituting from equation 2 into equation 8 yields:

$$
\dot{V}=r^{T}(y \phi-\tau)+r^{T}\left(\frac{1}{2} \dot{m}-b\right) r+\tilde{\phi}^{T} \gamma^{-1} \tilde{\dot{\phi}}
$$

Knowing that $\left(\frac{1}{2} \dot{m}-b\right)$ yields:

$$
\dot{V}=r^{T}(y \phi-\tau)+\tilde{\phi}^{T} \gamma^{-1} \tilde{\dot{\phi}}-r^{T} b r
$$

Substituting from equation 5 into equation 10 yields:

$$
\dot{V}=-r^{T} k_{v} r+\tilde{\phi^{T}}\left(\gamma^{-1} \tilde{\dot{\phi}}+y^{T} r\right)-r^{T} b r
$$

Substituting from equation 6 into equation 11 yields:

$$
\dot{V}=-r^{T} k_{v} r-r^{T} b r<0
$$

$V$ is lower bounded by zero, $\dot{V}$ is negative semidefinite and $\ddot{V}$ is bounded so by Barbalat's Lemma

$$
\lim _{t \rightarrow \infty} \dot{v}=0
$$

By the Rayleigh-Ritz theorem, we can prove that

$$
\lim _{t \rightarrow \infty} r=0
$$

which means that

$$
\lim _{t \rightarrow \infty} e=0
$$




\section{$2.2 \quad$ Fuzzy logic}

In classical logic, decisions are binary: either true or false. This what distinguishes fuzzy logic from classical logic. In fuzzy logic, a decision can be both true and false at the same time with a certain degree of membership to each of these two beliefs. In the robotic rehabilitation applications, the classic control could be applied by defining a pain threshold for each case. This threshold must be defined by the physiotherapist at the start of the rehabilitation process. However, the system in this case is not intelligent because we can not reach this threshold due to other parameters other than pain. For example, for small angles, when the motor starts, it causes a default current peak. If the controller considers this current request as resistance from the patient and therefore an indication of the sensation of pain, the system must stop and the reached ROM is considered as a limit for this rehabilitation step. On the other hand, the sensation of pain should not stop the process if the range of motion has already been reached. Thus, a fuzzy logic controller in which the last ROM and the current are considered as two inputs is needed to provide an intelligent rehabilitation system.

Fuzzy logic is a rule-based decision-making method used for expert systems and process control. As an integrated development environment, LabVIEW uses the Labview PID and the fuzzy logic toolbox containing the fuzzy system designer and the fuzzy logic VIs to design and control fuzzy systems. While fuzzy systems consist of three main parts: linguistic variables, membership functions and rules. The first step in designing a fuzzy system with Fuzzy System Designer is to create the input and output linguistic variables for the system. The next step is to create a rule base. Indeed, the rules describe the relationships between the input and output linguistic variables according to their linguistic terms. The fuzzy rule base determines fuzzy output values based on the input values. In rehabilitation protocols, medical experts recommend the application of autonomous robotic rehabilitation systems with more sensitivity and intelligence so that the robot detects the pain felt by the patient and therefore the possibility of incorporating this parameter in the control approach of the rehabilitation process. Based on this approach, the robot can imitate the therapist's activities with intelligence and great performance.

First of all, we were assisted by a medical expert to define the basis of the rules based on his experience in rehabilitation protocols. Then, preliminary tests are run to check the designed rule base. A second series of tests is launched to improve the rule base based on feedback from the physiotherapist. The series of tests continued until the satisfactory conventional rehabilitation protocol was followed.

The proposed fuzzy logic system is shown in Figure 3. The carried out range of motion (ROM) and the measured current are the fuzzy logic system inputs while the pain estimation is the system output. In fact, the proposed fuzzy system uses the already achieved ROM and the resistance against rehabilitation to estimate the patient pain during the therapy process. Also, the developed fuzzy controller takes into account the current peak values often caused by patient sensation of pain. This makes the developed system a smart decision making system compared to on-off switching systems. The membership functions for both inputs and output are shown in figure 4. The Gaussian membership functions are found the best suitable type to be used in the implemented system.

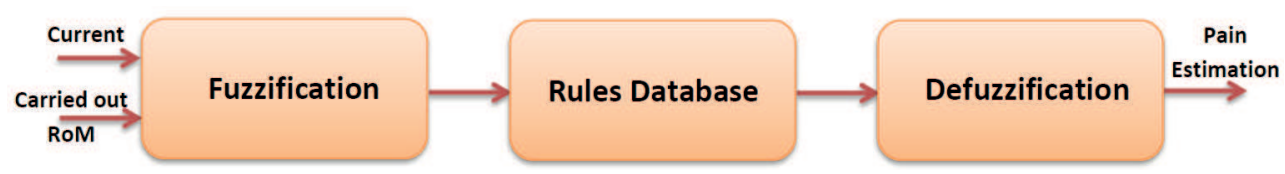

Figure 3: Fuzzy logic system

The proposed fuzzy logic system is implemented using the NI LabVIEW PID and Fuzzy Logic Toolkit. The Center of area (CoA) deffuzzification method is used. In order to unify the scale of the inputs, and the output of the fuzzy controller to be in the range from 0 to 10 , normalization is used using equation 13 .

$$
X_{N}=\frac{\left(X-X_{\min }\right)\left(X_{N \max }-X_{N \min }\right)}{\left(X_{\max }-X_{\min }\right)}+X_{\min }
$$

Where $X$ is the variable before normalization and its range is $\left[X_{\min }, X_{\max }\right] . X_{N}$ is the normalized 


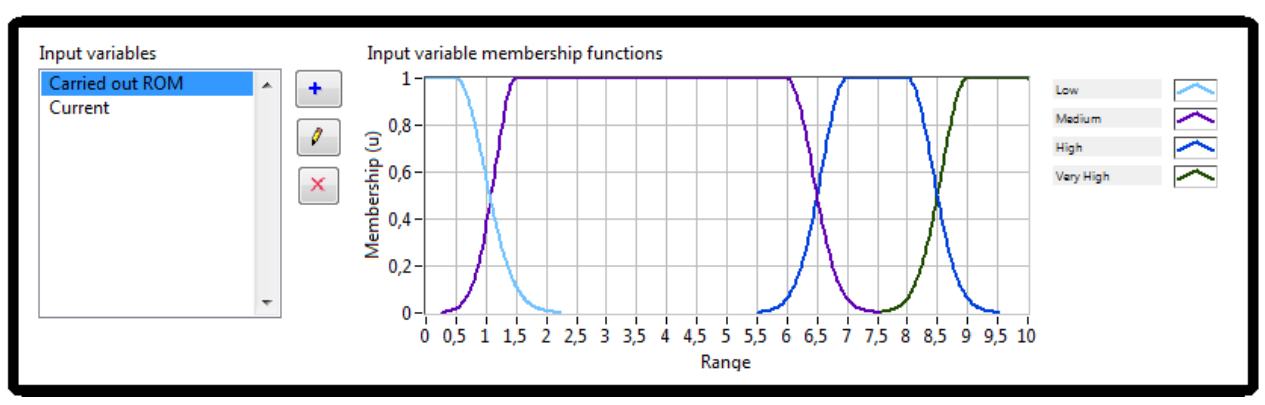

(a) Carried out ROM

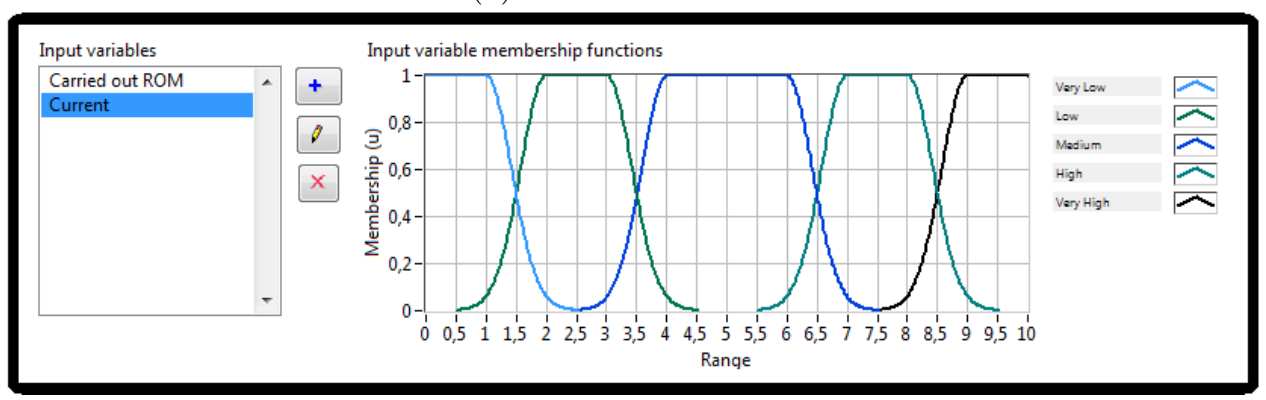

(b) Current

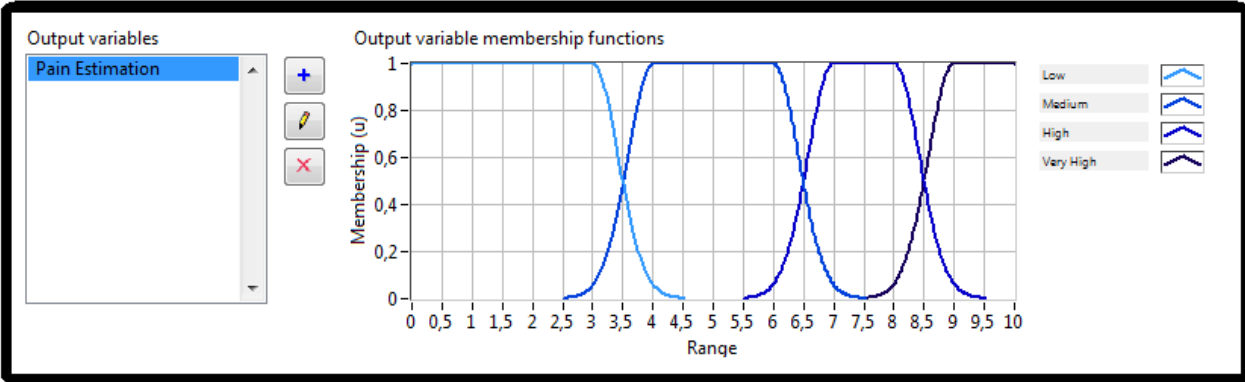

(c) Pain estimation

Figure 4: Membership functions

variable and its range is $\left[X_{N \min }, X_{N \max }\right]$.

The rule base that governs the operation of the proposed fuzzy logic is shown in Table 1. It is noted that for a small amplitude of movement, the system takes into account the peak current due to the starting of engine and thereafter, any request for current cannot reflect a feeling of pain. However, even in a real case of feeling pain often felt during a first rehabilitation session, this feeling in these very small ranges of movement is considered to be completely normal and can in no case cause an emergency for which the robot stops and deals with this case.

\section{Results and discussions}

In order to assess the validity and effectiveness of the proposed fuzzy architecture, some results and discussions are presented in this section. The developed wrist rehabilitation robot in this paper has many features that makes it best fit in home rehabilitation. It is light in weight, simple in its mechanical design, and easy to use. The detailed mechatronic design is presented in our previous work [7].

Fuzzy logic is a rule-based decision-making method used for expert systems and process control. As an integrated development environment, LabVIEW uses the Labview PID and the fuzzy logic toolbox containing the fuzzy system designer and the fuzzy logic VIs to design and control fuzzy systems. While fuzzy systems consist of three main parts: linguistic variables, membership functions and rules. The first step in designing a fuzzy system with Fuzzy System Designer is to create the input and output linguistic variables for the system. The next step is to create a rule base. Indeed, the rules 
Table 1: Rule database

\begin{tabular}{|l|l|l|l|}
\hline Rule no. & Carried our RoM & Current & Pain estimation \\
\hline 1 & L & L & L \\
\hline 2 & L & M & L \\
\hline 3 & L & H & L \\
\hline 4 & L & V.H & L \\
\hline 5 & L & V.L & L \\
\hline 6 & M & V.L & L \\
\hline 7 & M & L & L \\
\hline 8 & M & M & M \\
\hline 9 & M & H & H \\
\hline 10 & M & V.H & V.H \\
\hline 11 & H & V.L & L \\
\hline 12 & H & L & L \\
\hline 13 & H & M & M \\
\hline 14 & H & H & H \\
\hline 15 & H & V.H & V.H \\
\hline 16 & V.H & V.L & L \\
\hline 17 & V.H & L & L \\
\hline 18 & V.H & M & M \\
\hline 19 & V.H & H & H \\
\hline 20 & V.H & V.H & V.H \\
\hline
\end{tabular}

describe the relationships between the input and output linguistic variables according to their linguistic terms. The fuzzy rule base determines fuzzy output values based on the input values. In rehabilitation protocols, medical experts recommend the application of autonomous robotic rehabilitation systems with more sensitivity and intelligence so that the robot detects the pain felt by the patient and therefore the possibility of incorporating this parameter in the control approach of the rehabilitation process. Based on this approach, the robot can imitate the therapist's activities with intelligence and great performance.

First of all, we were assisted by a medical expert to define the basis of the rules based on his experience in rehabilitation protocols. Then, preliminary tests are run to check the designed rule base. A second series of tests is launched to improve the rule base based on feedback from the physiotherapist. The series of tests continued until the satisfactory conventional rehabilitation protocol was followed.

Compared to recent works dealing with rehabilitation based on EMG feedback [15, 29, 30], the developed solution in this paper uses the vision-based solution to avoid several problems like the sensitivity of EMG signals to electrode placement, interference with signals from neighboring muscles and skin properties. The main drawback of the EMG developed methodology is the lack of EMG signals' normalization which is very necessary as the EMG signals are highly affected by muscle noise and fatigue. In addition, the EMG signals do not take into account the velocity, because the area required for electromyography electrodes is large. Compared to [37, 49], the proposed solution is remotely controlled via internet which in turn greatly affect the whole rehabilitation process and all stakeholders [27]. The tele rehabilitation systems will allow the therapists to remotely control and monitor their patients at home. These benefits include but not restricted to minimum hospital stays, decreasing cost, and increasing the level of care. In contrast of the devices presented in [4? ], the proposed solution responds to the pain felt by the patient and deemed unbearable. Based on the estimated pain and the last RoM, the fuzzy rules are defined to control the actions of the robot.

The proposed rehabilitation system also has a communication interface shown in figure 5 to be used by the therapist. As shown, the interface has tabs for setup and monitoring, real \& virtual environments, and data storage \& reporting. The setup and monitoring tab gives the therapist the ability to setup the therapy exercise and to video monitor patients during the rehabilitation exercises. Additionally, the interface guides the therapist to the appropriate gesture control position. The interface also has an invalid position alert to indicate that the therapist position can not be recognized by the Kinect camera. The database \& storage tab shown in figure 6 gives the capability of saving some useful information about the patient personal data, the exercises and patient progress report.

The video images at the patient side are displayed on the therapist computer. These images are first captured by the IP camera connected to a router. The therapist computer is connected to the router as well. The IP camera is connected to the LabVIEW interface using the multimedia for LabVIEW 


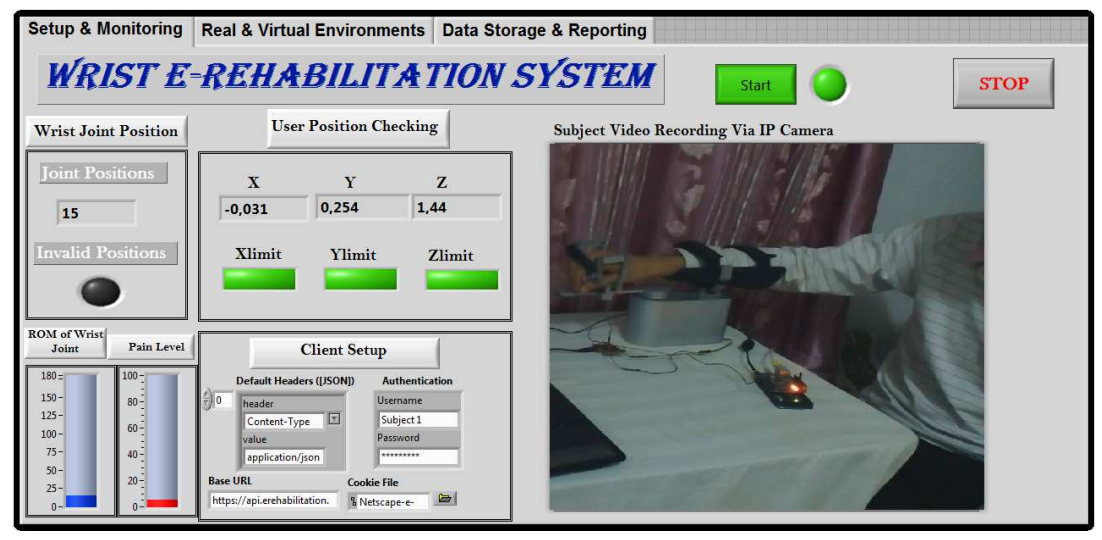

Figure 5: setup sequences

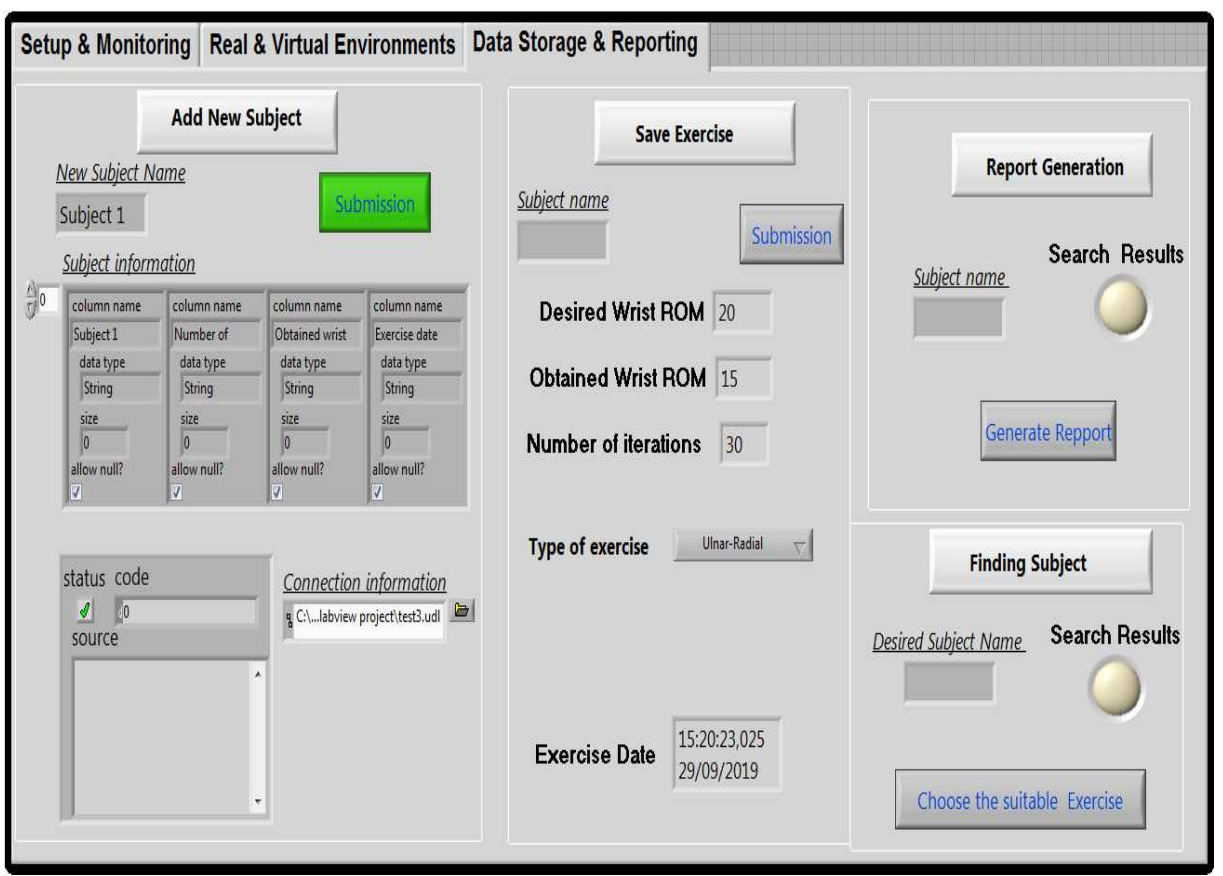

Figure 6: Data base interface

toolkit which allows streaming with different protocols and various codes. Figure 7 shows the real time streaming of the implemented system. The second screen shown in figure 7 represents the real and virtual environments. The patient's videos captured by the IP camera and the physiotherapist's videos captured by the 3D camera are transmitted and displayed on the control interface presented in figure 7. Therefore, this interface gathers the real-time acquisitions of the two cameras: the Kinect and the IP camera and also provides a virtual representation of the physiotherapist's arm. The idea is to display a virtual robot that translates the data acquired by the sensor and to map them into a $3 \mathrm{D}$ virtual environment in a pure emulation of the gesture.

To ensure patient safety, a pain level indicator is displayed in the physiotherapist interface to provide information about the patient's resistance. This resistance is fed into the fuzzy controller as an input. The output of the fuzzy controller will provide the therapist with a complete image about the patient and thus the best fit conditions for the therapy process.

Experiments are performed on a subject (a patient) with a wrist fracture, just one week after removal of the cast. The subject underwent a complete rehabilitation protocol to recover the abduction/adduction movements for 10 days. The exercise is controlled by the physiotherapist who tilts the hand in one direction maintaining the position for 5 seconds. The exercise is repeated for 3 sets of 10. The connected robot follows the physiotherapist's instructions. The first test showed that the subject had lost about $72 \%$ of the ROM of his wrist. Indeed, as shown in figure 6 (the second curve 


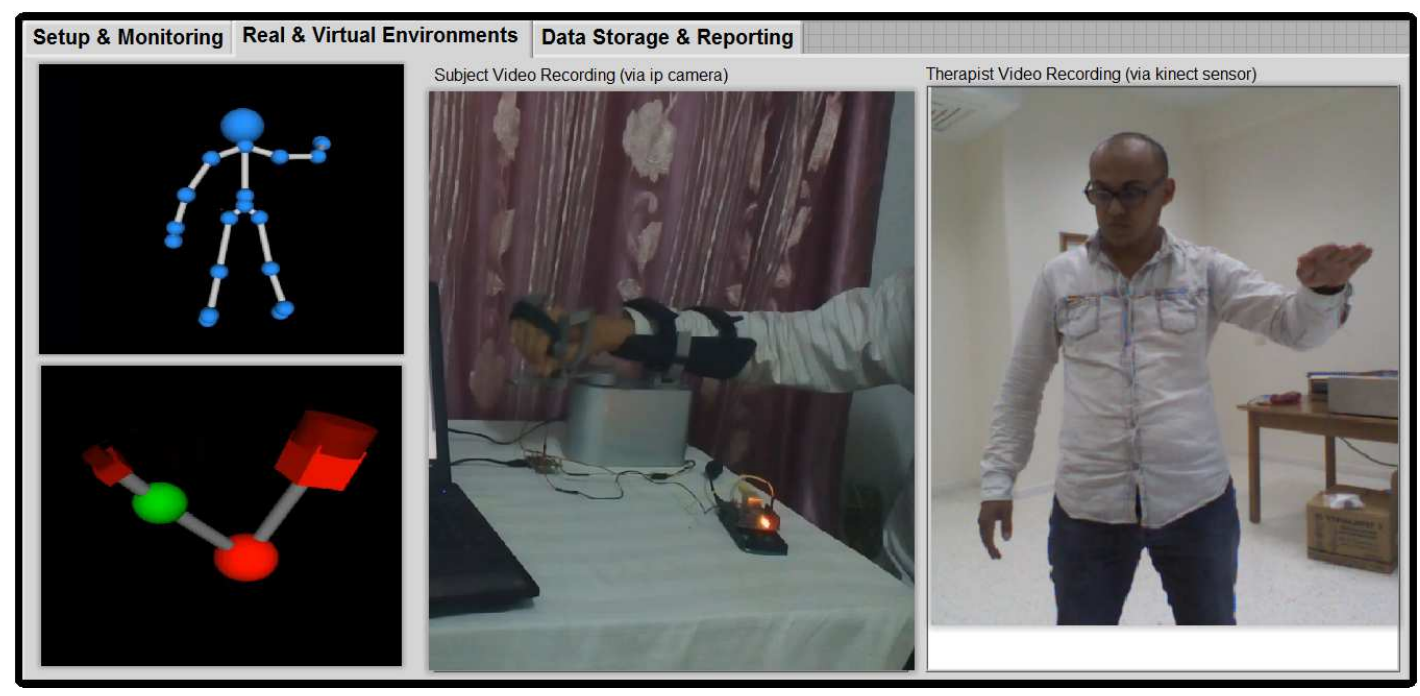

Figure 7: Real time streaming

Table 2: Wrist RoM for healthy subject

\begin{tabular}{ccccc}
\hline Mvt & Flexion & Extension & Radial & Ulnar \\
\hline RoM & $80-90$ & $70-90$ & 15 & $30-45$
\end{tabular}

illustrates the ROM as a function of the number of exercises), the subject finishes the first day with a ROM equal to 15 , which is $27 \%$ of the maximum ROM of a healthy subject (see table 2 ). In figure 8 , the first curve illustrates the progress of the ROM as a function of the number of iterations indicates the progression of the performance achieved by the subject on the last day. The subject reaches an amplitude of movement equal to 40 , which is $72 \%$ of the ROM reached by a healthy subject. The developed software displays 7.2 as a progression factor. This ratio is calculated based on the maximum ROM that a healthy subject can achieve, as shown in the table 2.

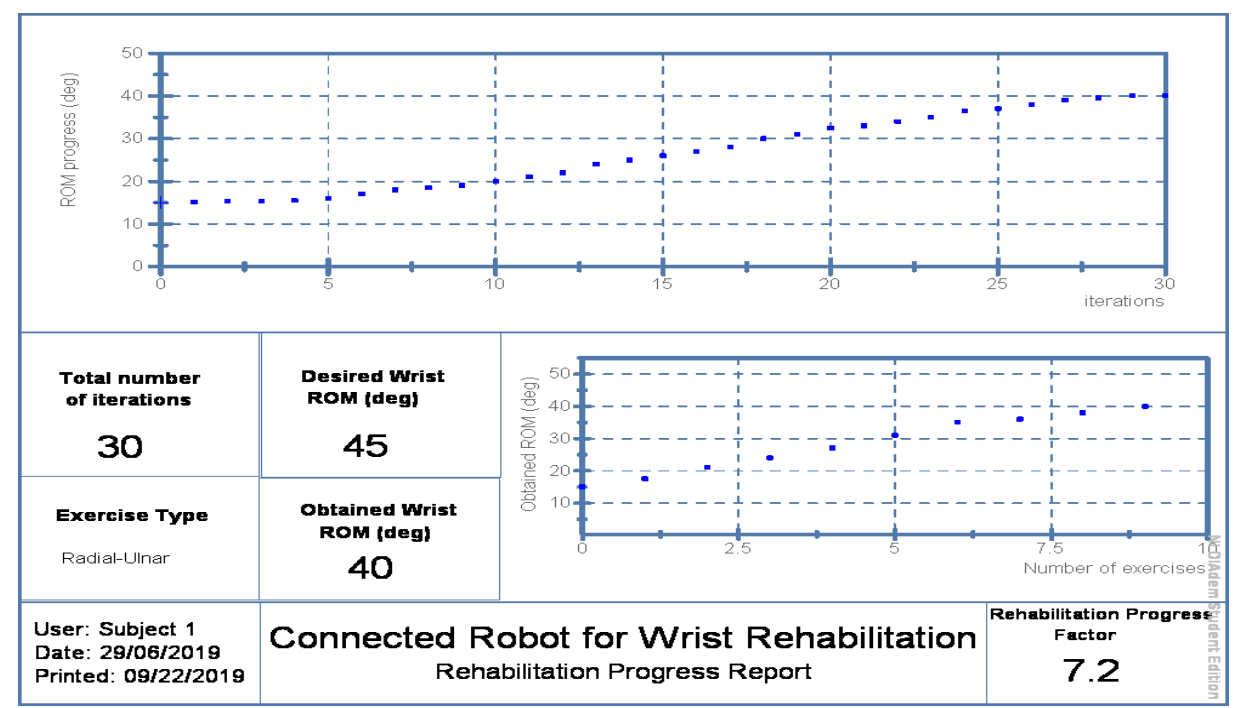

Figure 8: Final Report

Technically, the main limitation of the tele-rehabilitation is the communication delay inherent to the data processing and transmission on the network. The e-synchronization between the physiotherapist wrist movement and the action on the robot as well as the feedback displayed on the user interface can degrade the performance of the application. Our experiments show that the performance of the tele-rehabilitation technique decreases exponentially as the communication time delay increases. End 
to end delay can be expressed as follows:

$$
D_{\text {node }}=D_{\text {processing }}+D_{\text {queuing }}+D_{\text {transmission }}+D_{\text {propagation }}
$$

where

$$
D_{\text {transmission }}=\text { Segment Length } / \text { Rate }
$$

and

$$
D_{\text {queuing }}=D_{\text {transmission }} *(\text { Length of queue })
$$

To deal with the problem of delay, we have focused on the publishing intervals. Knowing that the delay of propagation can be approximated to half of the initial round trip time (IRTT/2). The round trip is the time taken by the signal between sent and acknowledgment. However, the IRTT is obtained by a simple ping from the PC to the IP address of the robot as shown in the figure 9 . The ping test gives $80 \mathrm{~ms}$. Therefore, messages are published every $200 \mathrm{~ms}$ and thus delays in programming and queuing are avoided.

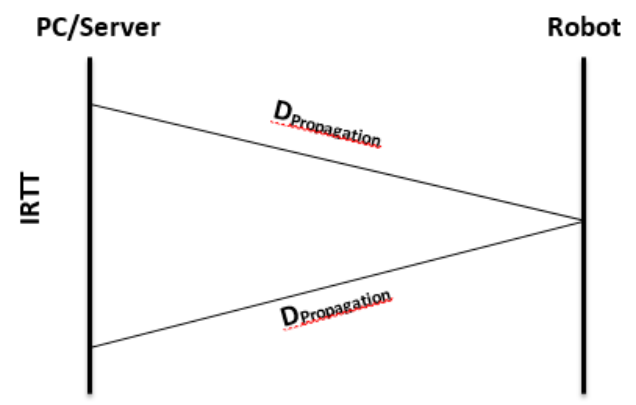

Figure 9: IRRT measurement

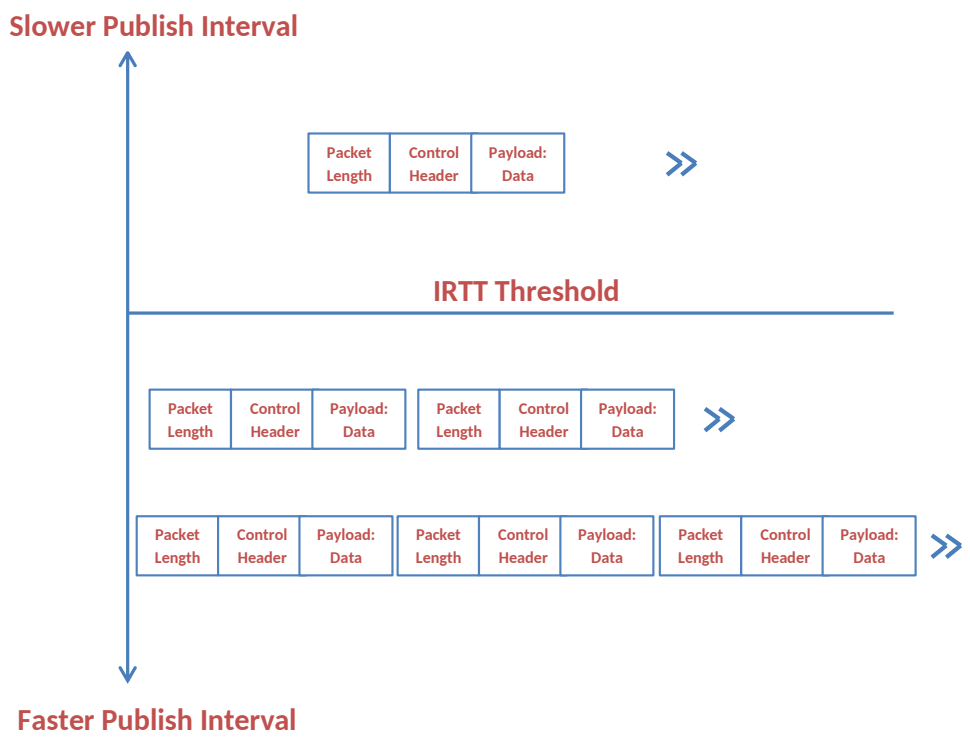

Figure 10: Influence of the publish interval on the queue

The choice of publish interval at least twice the IRTT cancels the propagation delay. The publish intervals can influence the queuing delay as shown in figure 10.

The developed code gives the user the opportunity to reset the TCP connection and to establish a new IRTT. The updated IRTT will be used on the calculation of the delay and consequently on the choice of the publishing intervals. 


\section{Conclusions}

Many benefits can be gained from using robots in rehabilitation starting from the repetition of exercises and ending with the complete monitoring of patients during the exercise protocol using the robot sensors. In this paper, an IoT architecture for rehabilitation robotic system is presented. The proposed architecture enables The physiotherapists to monitor and control patients from far. Fuzzy logic is adopted in the developed architecture to deal with patient pain which facilitates the control process specially for passive patients. The Kinect camera is employed which provides a low cost solution for $3 D$ vision-based systems. The proposed rehabilitation robotic system has interfaces for monitoring \& control, real \& virtual environments, and data storage and reporting.

\section{Acknowledgment}

The authors would like to acknowledge the support of the Deanship of Scientific Research at Prince Sattam Bin Abdulaziz University under the research project \# 2019/01/10761.

\section{References}

[1] Abdallah, I.B.; Bouteraa, Y.; Boucetta, R.; Chokri, R. (2015). Kinect-based Computed Torque Control for lynxmotion robotic arm, In 2015 7th International Conference on Modelling, Identification and Control (ICMIC), 1-6, 2015.

[2] Abdallah, I.B.; Bouteraa, Y.; Chokri, R. (2016). Kinect-based sliding mode control for Lynxmotion robotic arm, Advances in Human-Computer Interaction, 2016.

[3] Ali, A.; Ahmed, S.; Kadir, K. A. ; Karman, M.J.; Yarooq, R.S. (2018). Fuzzy PID controller for upper limb rehabilitation robotic system, 2018 IEEE International Conference on Innovative Research and Development (ICIRD), IEEE, 2018.

[4] Ates, S.; Haarman, C.; Stienen, A. (2017). SCRIPT passive orthosis: design of interactive hand and wrist exoskeleton for rehabilitation at home after stroke, Autonomous Robots, 41(3), 711-723, 2017.

[5] Bouteraa, Y.; Abdallah, I.B. (2016). Exoskeleton robots for upper-limb rehabilitation, 201613 th International Multi-Conference on Systems, Signals \& Devices (SSD), IEEE, 2016.

[6] Bouteraa, Y.; Abdallah, I.B. (2017). A gesture-based telemanipulation control for a robotic arm with biofeedback-based grasp, Industrial Robot: An International Journal, 44(5), 575-587, 2017.

[7] Bouteraa, Y.; Abdallah, I.B. ; chokri, R. (2016). Design of smart robot for wrist rehabilitation, International journal of smart sensing and intelligent systems, 9(2), 1029-1053, 2016.

[8] Bouteraa, Y; Abdallah, I.B.; Elmogy, A. (2019). Training of Hand Rehabilitation Using Low Cost Exoskeleton and Vision-Based Game Interface, Journal of Intelligent \& Robotic Systems, 96, 31-47,2019.

[9] Bower, K.J.; Louie, J.; Landesrocha, Y. et al. (2015), Clinical feasibility of interactive motioncontrolled games for stroke rehabilitation, Journal of NeuroEngineering and Rehabilitation, 12(63), 2015.

[10] Ding, I.; Chang, C. (2015). Eigenspace-based Method with a User Adaptation Scheme for Human Gesture Recognition by Using Kinect 3D Data, Applied Mathematical Modelling, Elsevier, 2015, 39(19), 5769-5777, 2015.

[11] Du, G.; Zhang, P. (2014). Markerless human-robot interface for dual robot manipulators using Kinect sensor, Robotics and Computer-Integrated Manufacturing, Elsevier, 30, 150-159, 2014. 
[12] Dzitac, I.; Filip, F.G.; Manolescu, M.J. (2017). Fuzzy logic is not fuzzy: World-renowned computer scientist Lotfi A. Zadeh, International Journal of Computers Communications \& Control, 12(6), 748-789, 2017.

[13] Dzitac, S.; Felea, I.; Dzitac, I. et al. (2008). An application of neuro-fuzzy modelling to prediction of some incidence in an electrical energy distribution center, International Journal of Computers Communications \& Control, 3(S), 287-292, 2008.

[14] Dzitac, S.; Vesselenyi, T.; Popper, L. et al. (2010). Fuzzy Algorithm for Human Drowsiness Detection Devices, Studies in Informatics and Control, 19(4), 419-426, 2010.

[15] Flores, A.; Verdezoto, G.; Tobar, L.; Iglesias, I.; Guerra, S. (2019). Angle Estimation of Wrist Movements Through Surface EMG Signals, In 2019 IEEE Fourth Ecuador Technical Chapters Meeting (ETCM), IEEE, 1-6, 2019.

[16] Gabriel, A. O.; Colgate, J.; Peshkin, M.; Goswami, A. (2016). Active Impedance Control of a lower limb exoskeleton to assist sit-to-stand movement, In Robotics and Automation (ICRA), 2016 IEEE International Conference on, 3530-3536, 2016.

[17] Galna, B.; Barry, G.; Jackson, D.; Mhiripiri, D.; Olivier, P.; Rochester, L. (2014). Accuracy of the Microsoft Kinect sensor for measuring movement in people with Parkinson's disease, Gait \& Posture, Elsevier, 39(4), 1062-1068, 2014.

[18] Gilzsolt, D.; Johanyak, C.; Kovacs, T. (2018). Surrogate model based optimization of traffic lights cycles and green period ratios using microscopic simulation and fuzzy rule interpolation, International Journal of Artificial Intelligence, 16(1), 20-40, 2018.

[19] Guozheng, X.; Aiguo, S.; Huijun, L. (2011) , Control system design for an upper-limb rehabilitation robot, Advanced Robotics, 25(1), 229-251, 2011.

[20] Haidegger, T.; Levente, K.; Preitl, S.; Precup, R.-E.; Balazs, B.; Benyo, Z. (2011). Controller design solutions for long distance telesurgical applications, International Journal of Artificial Intelligence, 6(11), 48-71, 2011.

[21] Harischandra, P. D.; Abeykoon, A. (2019). Intelligent bimanual rehabilitation robot with fuzzy logic based adaptive assistance, International Journal of Intelligent Robotics and Applications, 3(1), 59-70, 2019.

[22] Hua, J.; He, L.L.; Kang, Z.Q.; Yan. K.D. (2019). A Force/Position Hybrid Controller for Rehabilitation Robot, International Journal of Computers Communications \& Control, 14(5), 615-628, 2019 .

[23] Ibanez, R.; Soria, A.; Teyseyre, A.; Campo, M. (2014). Easy gesture recognition for Kinect, Advances in Engineering Software, Elsevier, 67, 171-180, 2014.

[24] Kanwal, N.; Bostanci, E.; Currie, K.; Clark, A. (2016). A Navigation System for the Visually Impaired: A Fusion of Vision and Depth Sensor, Applied Bionics and Biomechanics, Article ID 479857, 2015.

[25] Keemink, A.; Kooij, H.; Stienen, A. (2018). Admittance control for physical human-robot interaction, International Journal of Robotics Research, 37(11), 1421-1444, 2018.

[26] Kim, H.; Kim, I. (2015). Dynamic Arm Gesture Recognition Using Spherical Angle Features and Hidden Markov Models, Advances in Human-Computer Interaction, Article ID 785349, 2015.

[27] Klersy, C.; De Silvestri, A.; Gabutti, G.; Raisaro, A.; Curti, M.; Regoli, F.; Auricchio, A. (2011), Economic impact of remote patient monitoring: an integrated economic model derived from a meta-analysis of randomized controlled trials in heart failure, Eur J Heart Fail, 13, 450-459, 2011. 
[28] Lin, B.; Chen, J. L.; Hsu, H. C. (2018). Novel Upper-Limb Rehabilitation System Based on Attention Technology for Post-Stroke Patients: A Preliminary Study, IEEE Access, 6, 2720-2730, 2018.

[29] Liu, J.; Ren, Y.; Xu, D.; Kang, S.; Zhang, L. (2019). EMG-Based Real-Time Linear-Nonlinear Cascade Regression Decoding of Shoulder, Elbow and Wrist Movements in Able-Bodied Persons and Stroke Survivors, IEEE Transactions on Biomedical Engineering, 99(1), 2019.

[30] Lyu, M.; Chen, W.; Ding, X.; Wang, J.; Pei, Z.; Zhang, B. (2019). Development of an EMGControlled Knee Exoskeleton to Assist Home Rehabilitation in a Game Context, Frontiers in neurorobotics, 13(67), 2019.

[31] Marinho, M.; Adorno, B.; Harada, K. et al. (2019). A Unified Framework for the Teleoperation of Surgical Robots in Constrained Workspaces, In IEEE International Conference on Robotics and Automation, Montreal, Canada, 2721-2727, 2019.

[32] Mohammadi, E; Zohoor, H.; Khadem, M. (2016). Design and prototype of an active assistive exoskeletal robot for rehabilitation of elbow and wrist, Scientia Iranica Trans. B: Mechanical Engineering, 23, 998-1005, 2016.

[33] Munoz, C.; Vargas, F.; Curilem, M. et al. (2009). Fuzzy logic in genetic regulatory network models, International Journal of Computers Communications \& Control, 4(4), 363-373, 2009.

[34] Nikafrooz, N.; Mahjoob, M.; Tofigh, M. (2018). Design, Modeling, ; Fabrication of a 3-DOF Wrist Rehabilitation Robot, 2018 6th RSI International Conference on Robotics and Mechatronics (IcRoM), IEEE, 2018.

[35] Noje, D.; Tarca, R.; Dzitac, I.; Pop, N. (2019). IoT Devices Signals Processing based on Multidimensional Shepard Local Approximation Operators in Riesz MV-algebras, International Journal of Computers Communications \& Control, 14(1), 56-62, 2019.

[36] Noje, D.; Dzitac, I.; Pop, N.; Tarca, R.(2020). IoT Devices Signals Processing Based on Shepard Local Approximation Operators Defined in Riesz MV-Algebras, Informatica, 31(1), 131-142, 2020.

[37] Peng, L.; Hou, Z.; Peng, L.; Luo, L.; Wang, W. (2017) . Robot assisted rehabilitation of the arm after stroke: prototype design and clinical evaluation, Science China Information Sciences, 60(7), 2017.

[38] Pham, H.; Le, T.; Vuillerme, N. (2016). Real-Time Obstacle Detection System in Indoor Environment for the Visually Impaired Using Microsoft Kinect Sensor, Journal of Sensors, 2016.

[39] Precup, R.-E.; Tomescu, M.L.; Dragos, C.-A. (2014). Stabilization of Rossler chaotic dynamical system using fuzzy logic control algorithm, International Journal of General Systems, 43(5), 413-433, 2014.

[40] Simoens, P.; Dragone, M.; Saffiotti, A. (2018). The Internet of Robotic Things: A review of concept, added value and applications, International Journal of Advanced Robotic Systems, 1-11, 2018 .

[41] Sinasi, A. M.; Altas, I. H. (2017). Fuzzy logic based adaptive admittance control of a redundantly actuated ankle rehabilitation robot, Control Engineering Practice, 59,44-54, 2017.

[42] Strbac, M.; Kocovic, S.; Markovic, M.; Popovic, D. (2014), Microsoft Kinect-Based Artificial Perception System for Control of Functional Electrical Stimulation Assisted Grasping, BioMed Research International, Article ID 740469, 2014.

[43] Takacs, A.; Kovacs, L.; Rudas, I. et al. (2015). Models for force control in telesurgical robot systems, Acta Polytechnica Hungarica, 12(8), 95-114, 2015. 
[44] Tsarouchi, P.; Makris, S.; Chryssolouris, G. (2016), Human-robot interaction review and challenges on task planning and programming, International Journal of Computer Integrated Manufacturing, 29(8), 916-931, 2016.

[45] Tucan, P.; Gherman, B. ; Major, K. et al. (2020). Fuzzy logic-based risk assessment of a parallel robot for elbow and wrist rehabilitation, International Journal of Environmental Research and Public Health, 17(2), 654, 2020.

[46] Tuvshinjargal, D.; Dorj, B.; Lee, D. (2015). Hybrid Motion Planning Method for Autonomous Robots Using Kinect Based Sensor Fusion and Virtual Plane Approach in Dynamic Environments, Journal of Sensors, Article ID 471052, 2015.

[47] Vermesan, O.; Friess, P. (2014). Internet of Things-From Research and Innovation to Market Deployment, Aalborg, Denmark: River Publishers, 2014.

[48] Wang, C.; Peng, L.; Hou, Z.G. et al. (2019). A Novel Assist-As-Needed Controller Based on FuzzyLogic Inference and Human Impedance Identification for Upper-Limb Rehabilitation, 2019 IEEE Symposium Series on Computational Intelligence (SSCI), IEEE, 1133-1139, 2019.

[49] Wang, Y.; Xu, Q. (2019). Design of a New Wrist Rehabilitation Robot Based on Soft Fluidic Muscle, 2019 IEEE/ASME International Conference on Advanced Intelligent Mechatronics (AIM), IEEE, 595-600, 2019.

[50] Xu, T.; Jia, S.; Dong, Z.; Li, X. (2015). Obstacles Regions 3D-Perception Method for Mobile Robots Based on Visual Saliency. Journal of Robotics, Article ID 720174, 2015.

[51] Zihao, X.; Weiqun, W.; Zeng-Guang, H.; Xiaoming, L.; Xu, L. (2017). Dynamic model based fuzzy-impedance interaction control for rehabilitation robots, 2017 IEEE International Conference on Robotics and Biomimetics (ROBIO), IEEE, 1583-1588, 2017.

\section{(c) (i) (8)}

Copyright (C)2020 by the authors. Licensee Agora University, Oradea, Romania.

This is an open access article distributed under the terms and conditions of the Creative Commons Attribution-NonCommercial 4.0 International License.

Journal's webpage: http://univagora.ro/jour/index.php/ijccc/

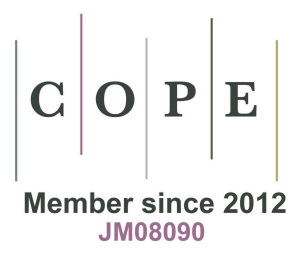

This journal is a member of, and subscribes to the principles of,

the Committee on Publication Ethics (COPE).

https://publicationethics.org/members/international-journal-computers-communications-and-control

Cite this paper as:

Bouteraa, Y.; Abdallah, I.B.; Elmogy, A.; Ibrahim, A.; Tariq, U.; Ahmad, T. (2020). A Fuzzy Logic Architecture for Rehabilitation Robotic Systems, International Journal of Computers Communications \& Control, 15(4), 3814, 2020.

https://doi.org/10.15837/ijccc.2020.4.3814 